\title{
Triple Therapy with Ecabet Sodium, Amoxicillin and Lansoprazole for 2 Weeks as the Rescue Regimen for $\boldsymbol{H}$. pylori Infection
}

\author{
Takahisa Furuta ${ }^{1}$, Mototsugu Kato ${ }^{2}$, Mitsushige Sugimoto ${ }^{1}$, Makoto Sasaki ${ }^{3}$, \\ Toshiro Kamoshida ${ }^{4}$, Kouichi Furukawa ${ }^{5}$, Tomoki Inaba ${ }^{6}$, Takashige Tomita ${ }^{7}$, \\ Takayuki Shirai ${ }^{8}$, Naoki Ishii ${ }^{9}$, Hideyuki Nomura ${ }^{10}$, Yoshitaka Konda ${ }^{11}$, \\ Masahiro Asaka ${ }^{12}$ and the JAPAN GAST STUDY GROUP
}

\begin{abstract}
Background/Aim Ecabet sodium has an anti-H. pylori effect. We assessed the efficacy of ecabet sodium in the rescue therapy for the eradication of $H$. pylori.

Methods A total of 74 patients with failed eradication of $H$. pylori after triple therapy with lansoprazole 30 $\mathrm{mg}$ bid, amoxicillin $750 \mathrm{mg}$ bid and clarithromycin $200 \mathrm{mg}$ bid were enrolled. They were randomly assigned to the three treatment groups as follows: LAC, lansoprazole $30 \mathrm{mg}+$ amoxicillin $750 \mathrm{mg}+$ clarithromycin $200 \mathrm{mg}$ bid for 1 week; LAC2E, lansoprazole $30 \mathrm{mg}$ bid + amoxicillin $750 \mathrm{mg}$ bid + clarithromycin $200 \mathrm{mg}$ bid + ecabet sodium $2 \mathrm{~g}$ bid for 1 week; and LA2E, lansoprazole $30 \mathrm{mg}$ bid + amoxicillin $750 \mathrm{mg}$ bid + ecabet sodium $2 \mathrm{~g}$ bid for 2 weeks. Eradication of $H$. pylori was assessed by the 13C-urea breath test after treatment.

Results Eradication rates in intention-to-treat and per-protocol analyses were $20.0 \%$ (95\% CI: 6.8-40.7) and $20.0 \%(6.8-40.7)$ with LAC, respectively, and $16.0 \%$ (4.5-36.1) and 17.4\% (5.0-38.8) with LAC2E. In contrast, respective rates with LA2E were $75 \%(53.3-90.2)$ and $85.7 \%$ (63.7-97.0), which were significantly higher than those with LAC ( $<<0.001$ for both ITT and PP) and LAC2E ( $<<0.001$ for both ITT and PP).

Conclusion Triple therapy with ecabet sodium, lansoprazole and amoxicillin for 2 weeks was effective as the rescue therapy after failure of the standard clarithromycin-based regimen.
\end{abstract}

Key words: Ecabet sodium, H. pylori, eradication, resistance, clarithromycin, metronidazole

(Intern Med 50: 369-374, 2011)

(DOI: 10.2169/internalmedicine.50.4305)

\section{Introduction}

Eradication of Helicobacter pylori (H. pylori) infection is now conducted for a variety of upper gastrointestinal tract disorders, including peptic ulcer and gastric mucosa- associated lymphoid tissue lymphoma (MALToma), and after endoscopic resection of gastric cancer in the early stage (1-5). The current standard first-line regimen for the eradication of $H$. pylori in Japan is the triple regimen with a proton pump inhibitor (PPI), amoxicillin and clarithromycin (6), while the standard second-line therapy consists of a

\footnotetext{
${ }^{1}$ Center for Clinical Research, Hamamatsu University School of Medicine, Japan, ${ }^{2}$ Division of Endoscopy, Hokkaido University Hospital, Japan, ${ }^{3}$ Department of Gastroenterology, Aichi Medical University School of Medicine, Japan, ${ }^{4}$ Department of Internal Medicine, Hitachi General Hospital, Japan, ${ }^{5}$ Department of Gastroenterology, Niigata City General Hospital, Japan, ${ }^{6}$ Department of Gastroenterology, Kagawa Prefectural Central Hospital, Japan, ${ }^{7}$ Department of Gastroenterology, Toshiba General Hospital, Japan, ${ }^{8}$ Department of Internal Medicine, Tokai University School of Medicine, Japan, ${ }^{9}$ Department of Gastroenterology (Gastroenterology Center), St. Luke's International Hospital, Japan, ${ }^{10}$ The Center for Liver Disease, Shin-Kokura Hospital, Japan, ${ }^{11}$ Department of Gastroenterology, Higashiyama Takeda Hospital, Japan and ${ }^{12}$ Department of Gastroenterology, Hokkaido University Graduate School of Medicine, Japan
}

Received for publication August 1, 2010; Accepted for publication November 16, 2010

Correspondence to Dr. Takahisa Furuta, furuta@hama-med.ac.jp 
Table 1. Demographic Clinical Characteristics of Patients Enrolled to the Study

\begin{tabular}{lllll}
\hline & LAC & LAC2E & LA2E & p value \\
\hline Number & 25 & 25 & 24 & \\
\hline Age, y \pm SE & $52.7 \pm 2.4$ & $52.8 \pm 2.4$ & $54.0 \pm 2.4$ & $>0.2^{1)}$ \\
Gender (male/female) & $14 / 11$ & $15 / 10$ & $15 / 9$ & $>0.2^{2)}$ \\
Smoking/non-smoking & $10 / 15$ & $10 / 15$ & $9 / 15$ & $>0.2^{2)}$ \\
GU/DU/GDU/gastritis & $14 / 9 / 1 / 1$ & $10 / 9 / 4 / 2$ & $15 / 7 / 2 / 0$ & $>0.2^{2)}$ \\
CAM-S/CAM-R/unknown & $6 / 15 / 4$ & $1 / 16 / 8$ & $2 / 8 / 14$ & $>0.2^{2)}$ \\
MNZ-S/MNZ-R/unknown & $11 / 5 / 9$ & $9 / 3 / 13$ & $10 / 4 / 10$ & $>0.2^{2)}$ \\
\hline
\end{tabular}

1) one-way ANOVA

2) Fisher's exact test

Abbreviations: $\mathrm{GU}=$ gastric ulcer, $\mathrm{DU}=$ duodenal ulcer, $\mathrm{GDU}=$ gastroduodenal ulcer, $\mathrm{LAC}=$ lansoprazole, amoxicillin and clarithromycin for 1 week, LAC2E = lansoprazole, amoxicillin, clarithromycin and ecabet sodium for 1 week

$\mathrm{LA} 2 \mathrm{E}=$ lansoprazole, amoxicillin and ecabet sodium for 2 weeks

CAM-S-clarithromycin-sensitive strain of $H$. pylori; CAM-R = clarithromycin-resistant strain of $H$.

pylori; MNZ-S = metronidazole-sensitive strain of $H$. pylori; MNZ-R = metronidazole-resistant strain of H. pylori.

PPI, amoxicillin and metronidazole (7).

An increase in the incidence of clarithromycin-resistant strains of $H$. pylori (8), which currently stands at approximately $30 \%$ or higher in Japan (9), has resulted in a decrease in the eradication rate of first-line treatment with a PPI, amoxicillin and clarithromycin in Japan to around $70 \%$ (9). Further, while the metronidazole-based second line therapy achieves eradication rates of approximately $90 \%$ (7) (10), the incidence of metronidazole resistantstrains is also increasing (11). Development of alternative rescue regimens with efficacy against strains resistant to clarithromycin and metronidazole is of clinical importance.

Ecabet sodium, developed as a gastric mucosal defensive agent for the treatment of peptic ulcer, has an anti-pepsin effect and induces prostaglandins in gastric mucosa (12-14). Ecabet sodium inhibits the urease activity of $H$. pylori and the growth of $H$. pylori in acidic conditions (15) (16). This anti-bacterial effect of ecabet sodium is observed even in strains resistant to clarithromycin and metronidazole (16).

Here, we investigated the usefulness of ecabet sodium in eradication therapy for $H$. pylori in patients in whom the initial therapy with lansoprazole, amoxicillin and clarithromycin failed.

\section{Materials and Methods}

This multi-center randomized controlled study at 11 hospitals in Japan was performed from 2005 to 2008. The protocol was approved in advance by the institutional review board (IRB) of each hospital.

\section{Patients}

Patients with peptic ulcer or gastritis for whom eradication of $H$. pylori failed on initial therapy with a triple regimen consisting of lansoprazole $30 \mathrm{mg}$ bid, amoxicillin 750 $\mathrm{mg}$ bid and clarithromycin $200 \mathrm{mg}$ bid for 1 week, the standard first-line therapy in Japan, were invited to participate in the study. Eradication failure was diagnosed based on a positive result in at least one of the following tests: rapid urease test, ${ }^{13} \mathrm{C}$-urea breath test, culture, or histology after eradication therapy.

Written informed consent was obtained from each patient before enrollment to the study. Exclusion criteria were age $<20$ or $>80$ years; use of antimicrobial agents within 3 months of the study; use of a PPI within 2 months of the study; two or more previous eradication failures; use of steroid or NSAIDs; severe general condition, such as renal insufficiency or liver dysfunction; unhealed ulcer; history of gastrectomy; inability to undergo repeated eradication therapy; allergy to agents used in this study; and serious complications considered to prevent completion of the study. Demographic characteristics of the subjects are summarized in Table 1. Subjects with compliance of less than $100 \%$ were considered to have dropped out.

\section{Eradication regimens and determination of success or failure of eradication}

A total of 74 eligible subjects were randomly assigned to the three treatment groups as follows: LAC, lansoprazole 30 $\mathrm{mg}$ bid + amoxicillin $750 \mathrm{mg}$ bid + clarithromycin $200 \mathrm{mg}$ bid for 1 week; LAC2E, lansoprazole $30 \mathrm{mg}$ bid + amoxicillin $750 \mathrm{mg}$ bid + clarithromycin $200 \mathrm{mg}$ bid + ecabet sodium $2 \mathrm{~g}$ bid for 1 week; and LA2E, lansoprazole $30 \mathrm{mg}$ bid + amoxicillin $750 \mathrm{mg}$ bid + ecabet sodium $2 \mathrm{~g}$ bid for 2 weeks. Eradication of $H$. pylori was assessed by $13 \mathrm{C}$-urea breath testing at 4 weeks after treatment.

Susceptibility to clarithromycin was assessed by either or both culture and measurement of A to $\mathrm{G}$ or $\mathrm{C}$ mutations at positions 2142 and 2143 of the 23S rRNA gene of $H$. pylori (17). Strains were considered resistant when the mean inhibitory concentration (MIC) for clarithromycin was ? 1 $\mu \mathrm{g} / \mathrm{mL}$ or mutation of $23 \mathrm{~S}$ rRNA was determined (18). Susceptibility to metronidazole was assessed by culture testing, and considered positive when MIC was $16 \geq \mu \mathrm{g} / \mathrm{mL}$. 


\section{Statistics}

Eradication rates were calculated based on intention-totreat (ITT) and per-protocol (PP) analyses and demonstrated with $95 \%$ confidential intervals. Comparison of eradication rates among regimen groups was assessed by Fisher's exact test. P-values less than $5 \%$ were considered statistically significant.

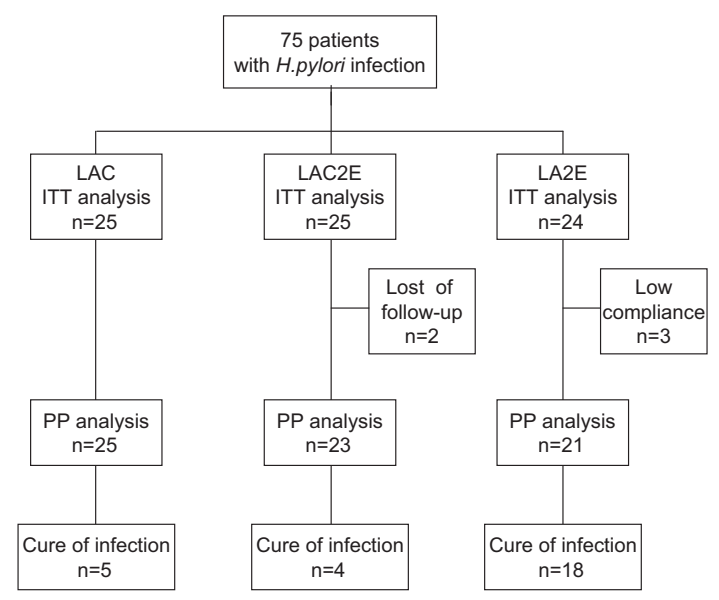

Figure 1. Flow of patients enrolled to the study.

\begin{tabular}{l} 
Results \\
\hline
\end{tabular}

Of 74 patients, 25 were assigned to the LAC, 25 to the LAC2E, and 24 to the LA2E groups (Fig. 1). There were no statistically significant differences between groups in mean age, male/female ratios, smoker/non-smoker ratios, or types of ulcer or gastritis (Table 1). Two patients who did not come to the hospital in the LAC2E and three whose compliance was less the $100 \%$ in the LA2E group were excluded from analysis.

Eradication rates by ITT analysis were $20.0 \%$ (95\% CI: $6.8 \%-40.7 \%$ ) in the LAC, $16.0 \%$ (95\% CI: $4.5 \%-36.1 \%$ ) in the LAC2E, and 75\% (95\% CI: $53.3 \%-90.2 \%$ ) in the LA2E groups. Respective rates by PP analysis were $20.0 \%(95 \%$ CI: $6.8 \%-40.7 \%$ ), $17.4 \%$ (95\% CI: $5.0 \%-38.8 \%$ ), and $85.7 \%$ (95\% CI: $63.7 \%-97.0 \%$ ). Eradication rate in the LA2E group was significantly higher than those in the LAC and LAC2E groups ( $<<0.001$ for both of LAC and LAC2E) (Table 2).

Susceptibility to clarithromycin was assessed in 59 of the 74 patients. Of these 59, $49(83 \%)$ were infected with clarithromycin-resistant strains of $H$. pylori. Eradication rates with these clarithromycin-resistant strains by the ITT analysis were $6.7 \%$ (95\% CI: $0.2 \%-31.9 \%$ ) in the LAC, $12.5 \%$ (95\% CI: $1.6 \%-38.3 \%$ ) in the LAC2E and $83.3 \%$ (95\% CI: $58.6 \%-96.4 \%$ ) in the LA2E groups (Table 3). Re-

Table 2. Eradication Rates of $\boldsymbol{H}$. pylori in the Three Groups

\begin{tabular}{cccc}
\hline & \multicolumn{2}{c}{$\begin{array}{l}\text { Eradication rate } \\
(\mathrm{n} / \mathrm{n})\end{array}$} & $95 \% \mathrm{CI}$ \\
\hline ITT & LAC & $20.0 \%(5 / 25)$ & $6.8 \%-40.7 \%$ \\
& LAC2E & $16.0 \%(4 / 25)$ & $4.5 \%-36.1 \%$ \\
& LA2E & $75.0 \%(18 / 24)^{\mathrm{a}, \mathrm{b}}$ & $53.3 \%-90.2 \%$ \\
\hline PP & LAC & $20.0 \%(5 / 25)$ & $6.8 \%-40.7 \%$ \\
& LAC2E & $17.4 \%(4 / 23)$ & $5.0 \%-38.8 \%$ \\
& LA2E & $85.7 \%(18 / 21)^{\mathrm{a}, \mathrm{b}}$ & $63.7 \%-97.0 \%$ \\
\hline
\end{tabular}

a: Significantly higher than LAC $(p<0.001), \quad$ b: significantly higher than LA2 CE $(p<0.001)$

LAC, lansoprazole, amoxicillin and clarithromycin, 1 week

LAC2E, lansoprazole, amoxicillin, clarithromycin and ecabet sodium, 1 week

LA2E, lansoprazole, amoxicillin and ecabet sodium, 2 weeks

ITT, Intention to treat; PP, per protocol.

Table 3. Eradication Rates in Patients Infected with Clarithromycinresistant Strains of $\boldsymbol{H}$. pylori

\begin{tabular}{lccc}
\hline ITT or PP & Regimen & Eradication rate $(\%)(\mathrm{n} / \mathrm{n})$ & $95 \%$ CI \\
\hline ITT & LAC & $6.7 \%(1 / 15)$ & $0.2 \%-31.9 \%$ \\
& LAC2E & $12.5 \%(2 / 16)$ & $1.6 \%-38.3 \%$ \\
& LA2E & $83.3 \%(15 / 18)^{\mathrm{a}, \mathrm{b}}$ & $58.6 \%-96.4 \%$ \\
\hline PP & LAC & $6.7 \%(1 / 15)$ & $0.2 \%-31.9 \%$ \\
& LAC2E & $12.5 \%(2 / 16)$ & $1.6 \%-38.3 \%$ \\
& LA2E & $88.2 \%(15 / 17)^{\mathrm{a}, \mathrm{b}}$ & $63.6 \%-98.5 \%$ \\
\hline
\end{tabular}

a: $p<0.001$ when compared with LAC, b: $p<0.001$ when compared with LAC2E

LAC, lansoprazole, amoxicillin and clarithromycin, 1 week

LAC2E, lansoprazole, amoxicillin, clarithromycin and ecabet sodium, 1 week

LA2E, lansoprazole, amoxicillin and ecabet sodium, 2 weeks

ITT, Intention to treat; PP, per protocol. 
spective rates by $\mathrm{PP}$ analysis were $6.7 \%$ (95\% CI: 0.2-31.9), $12.5 \%$ (95\% CI: $1.6-38.3$ ), and $88.2 \%$ (95\% CI: 63.6-98.5). The eradication rate in the LA2E group was significantly higher than those in the LAC and LAC2E groups on both ITT and PP analyses.

Susceptibility to metronidazole was assessed in 40 of the 74 patients. Of these $40,12(30 \%)$ were infected with metronidazole-resistant strains of $H$. pylori (Table 4), all of which were resistant to clarithromycin. In patients infected with metronidazole-sensitive strains, eradication was achieved in 3 of 11 in the LAC group and 1 of 9 in the LAC2E group. However, these regimens were unable to eradicate infection in patients with metronidazole-resistant strains. In contrast, the LA2E regimen was able to eradicate infection in 8 of 8 patients $(100 \%)$ infected with metronidazole-sensitive strains and 4 of 4 (100\%) infected with metronidazole-resistant strains of $\mathrm{H}$. pylori.

No adverse events were observed in the LAC group. In the LAC2E group, dysgeusia and hot flashes were observed in one patient each. In the LA2E group, loose stool was observed in two patients and diarrhea in two (Table 5). Of 2 diarrhea cases, one patient discontinued the treatment.

\section{Discussion}

In the present study, we compared the eradication rates obtained with three anti-H. pylori regimens, LAC, LAC2E and LA2E, in patients in whom eradication by the triple regimen with a PPI, clarithromycin and amoxicillin had failed. Results showed that the LA2E regimen may represent a candidate rescue regimen after the failure of eradication with the triple PPI/clarithromycin/amoxicillin therapy.

One of the major causes of eradication failure with triple $\mathrm{PPI} /$ clarithromycin/amoxicillin therapy is bacterial resistance

Table 4. Eradication Rates in Patients Infected with Metronidazole-sensitive and Resistant Strains of $\boldsymbol{H}$. pylori

\begin{tabular}{lcc}
\hline Regimens & Metronidazole-sensitive & Metronidazole-resistant \\
\hline LAC & $20.0(1 / 5)$ & $27.3(3 / 11)$ \\
LAC2E & $0.0(0 / 3)$ & $11.1(1 / 9)$ \\
LA2E & $100.0(4 / 4)$ & $100.0(8 / 8)$ \\
\hline LAC: lansoprazole, amoxicillin and clarithromycin, 1 week \\
LAC2E: lansoprazole, amoxicillin, clarithromycin and ecabet sodium, 1 week \\
LA2E: lansoprazole, amoxicillin and ecabet sodium, 2 weeks
\end{tabular}

to clarithromycin (6). The incidence of clarithromycinresistant strains in the present patients, all of whom had failed eradication by this therapy, was $83 \%$ (49/59). This finding indicates that rescue therapy should not be based around clarithromycin-containing regimens. Unfortunately, at the time the present study was started, metronidazole had yet to be permitted for use in Japan, and the same regimen as in initial therapy was repeated. This explains why our study regimens included LAC, which was also used as the first-line regimen. Recently, triple therapy consisting of a PPI, amoxicillin and metronidazole was approved as a second-line regimen for the eradication of $H$. pylori in Japan. As demonstrated in this study, however, the incidence of metronidazole-resistant strains of $H$. pylori is relatively high in patients who fail in the initial therapy, and several adverse events related to metronidazole have been reported (19) (20). These findings highlight the need for a variety of rescue strategies for patients with eradication failure. Recently, eradication with new regimens which include levofloxacin and rifabutin have been performed (21). However, adverse events related to these new regimens and drugs have not been fully elucidated.

Several reports have demonstrated that ecabet sodium used with any of several regimens increases the eradication rate of $H$. pylori infection: Ohkusa et al (22) reported that ecabet sodium increased the eradication rates of dual therapy with lansoprazole and amoxicillin from 58\% to 78\%; Kagaya et al (23) reported that this agent increased the eradication rate by dual lansoprazole/amoxicillin therapy from $43 \%$ to $79 \%$; and Kim et al (24) reported that it increased the eradication rate with triple PPI/amoxicillin/clarithromycin therapy from $78.8 \%$ to $88.6 \%$. In the present study, therefore, we examined the eradication rates of ecabet sodiumbased regimens, such as LAC2E for 1 week and LA2E for 2 weeks, in comparison with LAC for 1 week in patients in whom eradication of $H$. pylori with the triple PPI/amoxicillin/clarithromycin therapy had failed. Results showed no statistically significant differences in eradication rate between the LAC2E and LAC groups, whereas LA2E for 2 weeks yielded a markedly higher eradication rate than those with LAC2E and LAC.

In their study, Kim et al enrolled patients who had never undergone eradication therapy, while the present study patients had all failed eradication. This difference might, at least to some extent, explain the difference in results be-

Table 5. Adverse Events Observed in the Study Regimens (\%)

\begin{tabular}{lccc}
\hline & LAC $(\mathrm{n}=25)$ & LAC2E $(\mathrm{n}=25)$ & LA2E $(\mathrm{n}=24)$ \\
\hline Loose stool & $0(0.0)$ & $1(4.0)$ & $2(8.0)$ \\
Diarrhea & $0(0.0)$ & $0(0.0)$ & $2(8.0)$ \\
Dysgeusia & $0(0.0)$ & $1(4.0)$ & $0(0.0)$ \\
Hot flashes & $0(0.0)$ & $1(4.0)$ & $0(0.0)$ \\
\hline
\end{tabular}

LAC, lansoprazole, amoxicillin and clarithromycin, 1 week

LAC2E, lansoprazole, amoxicillin, clarithromycin and ecabet sodium, 1 week

LA2E, lansoprazole, amoxicillin and ecabet sodium, 2 weeks 
tween the two studies, (24). However, our present eradication rate by triple therapy with lansoprazole, amoxicillin and ecabet sodium for 2 weeks was $85 \%$, and $88.2 \%$ in those infected with clarithromycin-sensitive and -resistant strains of H. pylori, respectively. This regimen also attained eradication in patients infected with either metronidazole-resistant or metronidazole-sensitive strains. We therefore consider that ecabet sodium is effective against $H$. pylori strains irrespective of susceptibility to other antimicrobial agents, such as clarithromycin and metronidazole, when used with a PPI and amoxicillin for 2 weeks.

Although the bactericidal effect of ecabet sodium is not as strong as that of antibiotics, it potently inhibits the growth and urease activity of $H$. pylori. Interestingly, its bactericidal effect is enhanced in acidic conditions. We therefore consider that the effect of ecabet sodium is complementary to that of antibiotics, because the bioavailability of antibiotics is decreased in acidic conditions, whereas the anti-H. pylori effect of ecabet sodium is in contrast enhanced. One reason for eradication failure with a regimen consisting of a PPI and antibiotics is insufficient acid inhibition with a PPI. However, the bactericidal effect of ecabet sodium is increased when acid inhibition is insufficient. The addition of ecabet sodium to $H$. pylori eradication regimens theoretically increases eradication rates, particularly in those patients in whom the acid inhibition attained with a PPI is insufficient.

We assume that a second reason for the higher eradication with the LA2E regimen is related to the duration of the treatment period. Duration of most dual PPI/amoxicillin therapies that provided eradication rates higher than $80 \%$ were 2 weeks or longer (25) (26). Because amoxicillin is a time-dependent antibiotic (27) (28), further increases in eradication rates with amoxicillin-based therapy may require longer treatment periods.

Several limitations of our study warrant mention. First, the sample size was relatively small. However, the eradication rate with LA2E for 2 weeks was significantly higher than that with LAC and LAC2E for 1 week. Second, we did not measure the bactericidal effect of ecabet sodium in individual strains by culture testing. Thirdly, we did not test the eradication rate by LA for 2 weeks. Therefore, our study results could not demonstrate whether ecabet sodium really had an additional effect on the dual PPI/amoxicillin therapy. Together, the present results should thus be considered as providing preliminary guidance for future studies under a more appropriate design. Allowing for these limitations, the present results indicate that the triple therapy with lansoprazole, amoxicillin and ecabet sodium for 2 weeks is effective as a rescue regimen after the failure of eradication by the standard regimen.

\section{The authors state that they have no Conflict of Interest (COI).}

\section{Acknowledgement}

This study was supported by a grant-in aid from the Ministry of Education, Culture, Sports, Science and Technology of Japan
(20590718).

Abbreviations: LAC: lansoprazole, amoxicillin and clarithromycin for 1 week, LAC2E: lansoprazole, amoxicillin, clarithromycin and ecabet sodium for 1 week, LA2E: lansoprazole, amoxicillin and ecabet sodium for 2 weeks

\section{References}

1. Wotherspoon AC. Helicobacter pylori infection and gastric lymphoma. Br Med Bull 54: 79-85, 1998.

2. Uemura N, Okamoto S, Yamamoto $\mathrm{S}$, et al. Helicobacter pylori infection and the development of gastric cancer. N Engl J Med 345: 784-789, 2001.

3. Marshall BJ, Goodwin CS, Warren JR, et al. Prospective doubleblind trial of duodenal ulcer relapse after eradication of Campylobacter pylori. Lancet 2: 1437-1442, 1988.

4. Soll AH. Consensus conference. Medical treatment of peptic ulcer disease. Practice guidelines. Practice Parameters Committee of the American College of Gastroenterology. JAMA 275: 622-629, 1996.

5. Malfertheiner P, Megraud F, O'Morain C, et al. Current concepts in the management of Helicobacter pylori infection--the Maastricht 2-2000 Consensus Report. Aliment Pharmacol Ther 16: 167180, 2002.

6. Asaka M, Sugiyama T, Kato M, et al. A multicenter, double-blind study on triple therapy with lansoprazole, amoxicillin and clarithromycin for eradication of Helicobacter pylori in Japanese peptic ulcer patients. Helicobacter 6: 254-261, 2001.

7. Murakami K, Sato R, Okimoto T, et al. Efficacy of triple therapy comprising rabeprazole, amoxicillin and metronidazole for secondline Helicobacter pylori eradication in Japan, and the influence of metronidazole resistance. Aliment Pharmacol Ther 17: 119-123, 2003.

8. Tokunaga K, Tanaka A, Sugano H, Takahashi S. The present status and problems of Helicobacter pylori first-line eradication therapy. Nippon Rinsho 67: 2291-2296, 2009 (in Japanese).

9. Furuta T, Shirai N, Kodaira M, et al. Pharmacogenomics-based tailored versus standard therapeutic regimen for eradication of $H$. pylori. Clin Pharmacol Ther 81: 521-528, 2007.

10. Nagahara A, Miwa $H$, Kawabe $M$, et al. Second-line treatment for Helicobacter pylori infection in Japan: proton pump inhibitorbased amoxicillin and metronidazole regimen. J Gastroenterol 39: 1051-1055, 2004.

11. Koletzko S, Richy F, Bontems P, et al. Prospective multicentre study on antibiotic resistance of Helicobacter pylori strains obtained from children living in Europe. Gut 55: 1711-1716, 2006.

12. Ito $Y$, Nakamura S, Onoda Y, Sugawara $Y$, Takaiti O. Effects of the new anti-ulcer drug ecabet sodium (TA-2711) on pepsin activity. I. Inactivation of enzyme protein. Jpn J Pharmacol 62: 169174, 1993.

13. Kinoshita M, Yamasaki K, Kokusenya Y, Tamaki H. Relationship between gastroprotective effect of locally acting antiulcer agent ecabet sodium and its binding to gastric mucosa in rats. Comparison with sucralfate. Dig Dis Sci 40: 661-667, 1995.

14. Kinoshita M, Endo M, Yasoshima A, et al. Ecabet sodium, a novel locally-acting anti-ulcer agent, protects the integrity of the gastric mucosal gel layer from pepsin-induced disruption in the rat. Aliment Pharmacol Ther 13: 687-694, 1999.

15. Ito Y, Shibata K, Hongo A, Kinoshita M. Ecabet sodium, a locally acting antiulcer drug, inhibits urease activity of Helicobacter pylori. Eur J Pharmacol 345: 193-198, 1998.

16. Shibata K, Ito Y, Hongo A, Yasoshima A, Endo T, Ohashi M. Bacterial activity of a new antiulcer agent, ecabet sodium, against Helicobacter pylori under acidic conditions. Antimicrob Agents Chemother 39: 1295-1299, 1995. 
17. Furuta T, Soya Y, Sugimoto M, et al. Modified allele-specific primer-polymerase chain reaction method for analysis of susceptibility of Helicobacter pylori strains to clarithromycin. J Gastroenterol Hepatol 22: 1810-1815, 2007.

18. Adamek RJ, Suerbaum S, Pfaffenbach B, Opferkuch W. Primary and acquired Helicobacter pylori resistance to clarithromycin, metronidazole, and amoxicillin--influence on treatment outcome. Am J Gastroenterol 93: 386-389, 1998.

19. Knowles S, Choudhury T, Shear NH. Metronidazole hypersensitivity. Ann Pharmacother 28: 325-326, 1994.

20. Bradley WG, Karlsson IJ, Rassol CG. Metronidazole neuropathy. Br Med J 2: 610-611, 1977.

21. Gisbert JP, Morena F. Systematic review and meta-analysis: levofloxacin-based rescue regimens after Helicobacter pylori treatment failure. Aliment Pharmacol Ther 23: 35-44, 2006.

22. Ohkusa T, Takashimizu I, Fujiki K, et al. Prospective evaluation of a new anti-ulcer agent, ecabet sodium, for the treatment of Helicobacter pylori infection. Aliment Pharmacol Ther 12: 457461, 1998.

23. Kagaya H, Kato M, Komatsu YM, et al. High-dose ecabet sodium improves the eradication rate of Helicobacter pylori in dual therapy with lansoprazole and amoxicillin. Aliment Pharmacol Ther 14: 1523-1527, 2000.

24. Kim HW, Kim GH, Cheong JY, et al. H. pylori eradication: a randomized prospective study of triple therapy with or without ecabet sodium. World J Gastroenterol 14: 908-912, 2008.

25. Bayerdorffer E, Miehlke S, Mannes GA, et al. Double-blind trial of omeprazole and amoxicillin to cure Helicobacter pylori infection in patients with duodenal ulcers. Gastroenterology 108: 14121417, 1995.

26. Furuta T, Shirai N, Takashima M, et al. Effects of genotypic differences in CYP2C19 status on cure rates for Helicobacter pylori infection by dual therapy with rabeprazole plus amoxicillin. Pharmacogenetics 11: 341-348, 2001.

27. Craig WA. Basic pharmacodynamics of antibacterials with clinical applications to the use of beta-lactams, glycopeptides, and linezolid. Infect Dis Clin North Am 17: 479-501, 2003.

28. Craig WA. Proof of concept: performance testing in models. Clin Microbiol Infect 10 (Suppl 2): 12-17, 2004.

(C) 2011 The Japanese Society of Internal Medicine http://www.naika.or.jp/imindex.html 